\title{
Stability of bimaxillary surgery involving intraoral vertical ramus osteotomy with or without presurgical miniscrew-assisted rapid palatal expansion in adult patients with skeletal Class 111 malocclusion
}

\author{
Yoon-Soo Ahn ${ }^{\mathrm{a}}$ (1) \\ Sung-Hwan $\mathrm{Choi}^{\mathrm{a}, \mathrm{b}}$ (1) \\ Kee-Joon Lee ${ }^{\mathrm{a}}$ \\ Young-Soo Jung ${ }^{\mathrm{c}}$ \\ Hyoung-Seon Baik ${ }^{\mathrm{a}}$ \\ Hyung-Seog $\mathrm{Yu}^{\mathrm{a}}$ (1)
}

${ }^{a}$ Department of Orthodontics, Institute of Craniofacial Deformity, Yonsei University College of Dentistry, Seoul, Korea

${ }^{\mathrm{b} B K 21}$ PLUS Project, Yonsei University College of Dentistry, Seoul, Korea

'Department of Oral and Maxillofacial Surgery, Oral Science Research Center, Yonsei University College of Dentistry, Seoul, Korea

\begin{abstract}
Objective: The aim of this study was to evaluate the stability of bimaxillary surgery involving bilateral intraoral vertical ramus osteotomy performed with or without presurgical miniscrew-assisted rapid palatal expansion (MARPE) in adult patients with skeletal Class 111 malocclusion. Methods: A total of 40 adult patients with skeletal Class 111 malocclusion were retrospectively divided into two groups ( $\mathrm{n}=20$ each) according to the use of MARPE for the correction of transverse maxillomandibular discrepancy during presurgical orthodontic treatment. Serial lateral cephalograms and dental casts were analyzed until 6 months after surgery. Results: Before presurgical orthodontic treatment, there was no significant differences in terms of sex and age between groups. However, the difference of approximately $3.1 \mathrm{~mm}$ in the maxillomandibular intermolar width was statistically significant $(p<0.001)$. Two days after surgery, the mandible had moved backward and upward without any significant intergroup difference. Six months after surgery, the maxillary intercanine $(2.7 \pm 2.1 \mathrm{~mm})$, interpremolar $(3.6 \pm 2.4 \mathrm{~mm})$, and intermolar $(2.0 \pm 1.3 \mathrm{~mm})$ arch widths were significantly increased $(p<0.001)$ relative to the values before presurgical orthodontic treatment in the MARPE group; these widths were maintained or decreased in the control group. However, there was no significant difference in surgical changes and the postsurgical stability between the two groups. No significant correlations existed between the amount of maxillary expansion and postsurgical mandibular movement. Conclusions: MARPE is useful for stable and nonsurgical expansion of the maxilla in adult patients with skeletal Class 111 malocclusion who are scheduled for bimaxillary surgery.
\end{abstract}

[Korean J Orthod 2020;50(5):304-313]

Key words: Miniscrew-assisted rapid palatal expansion, Transverse maxillomandibular discrepancy, Skeletal Class 111 malocclusion, Stability

Received March 5, 2020; Revised April 3, 2020; Accepted April 25, 2020.

Corresponding author: Hyung-Seog Yu.

Professor, Department of Orthodontics, Institute of Craniofacial Deformity, Yonsei University College of Dentistry, 50-1 Yonsei-ro, Seodaemun-gu, Seoul 03722, Korea.

Tel +82-2-2228-3104 e-mail yumichael@yuhs.ac

Yoon-Soo Ahn and Sung-Hwan Choi contributed equally to this study.

How to cite this article: Ahn YS, Choi SH, Lee KJ, Jung YS, Baik HS, Yu HS. Stability of bimaxillary surgery involving intraoral vertical ramus osteotomy with or without presurgical miniscrew-assisted rapid palatal expansion in adult patients with skeletal Class 111 malocclusion. Korean J Orthod 2020;50:304-313.

(C) 2020 The Korean Association of Orthodontists.

This is an Open Access article distributed under the terms of the Creative Commons Attribution Non-Commercial License (http://creativecommons.org/licenses/by-nc/4.0) which permits unrestricted non-commercial use, distribution, and reproduction in any medium, provided the original work is properly cited. 


\section{INTRODUCTION}

Some adult patients with skeletal Class 111 malocclusion exhibit severe maxillomandibular anteroposterior discrepancy with transverse maxillary deficiency, which renders the treatment more challenging. ${ }^{1}$ In such cases, the clinician can address the transverse discrepancy using both surgical and nonsurgical methods.

Surgical expansion can be achieved with segmental maxillary osteotomy, which can be performed simultaneously with bimaxillary surgery in the operating room. However, it produces inaccurate and unstable outcomes. $^{2,3}$ Consequently, surgically-assisted rapid palatal expansion (SARPE) has been widely performed, although clinicians and patients might be concerned about additional issues such as hospitalization, attendant morbidity, increased cost, and surgical complications. ${ }^{4,5}$

For nonsurgical expansion, clinicians can decide to extract the maxillary premolars for relative expansion of the maxillary arch during presurgical orthodontic treatment. ${ }^{6}$ However, premolar extraction loses its applicability if the maxillary arch shows no or mild crowding. In addition, this modality lengthens the presurgical orthodontic treatment period, thus deteriorating the patient's quality of life. A transpalatal arch or conventional rapid palatal expansion (RPE) is generally not feasible in adults because of possible adverse effects such as buccal tipping, root resorption, and gingival recession around the anchor teeth. ${ }^{7}$

Recently, Lee et al. ${ }^{8}$ reported a successful clinical outcome for a patient who underwent orthognathic surgery and tooth-bone-borne RPE, which was assisted by four palatally placed orthodontic titanium miniscrews (i.e., miniscrew-assisted RPE [MARPE]). The miniscrews can reduce stress on the anchor teeth when the appliance expands the maxillary arch, thus reducing the side effects on the anchor teeth. ${ }^{9}$

Anterior and inferior movement of the maxilla and subsequent clockwise rotation of the mandible have been reported to occur with use of the aforementioned surgical and nonsurgical procedures for maxillary expansion. ${ }^{10-12}$ If transverse maxillary expansion can lead to such vertical and anteroposterior changes in the maxillomandibular complex, any relapse can have considerable effects on the skeletal changes after orthognathic surgery. In particular, when bimaxillary surgery such as bilateral intraoral vertical ramus osteotomy (IVRO) is performed, the mandible reportedly tends to undergo clockwise rotation due to muscular pull during the postoperative retention period. ${ }^{13}$ Even if slight maxillary contraction occurs, which is possible after MARPE, it may increase the probability of an anterior open bite after surgery. Moreover, maxillary constriction can lead to palatal inclination of the maxillary posterior teeth and an edge-to-edge bite between the posterior teeth on one or both sides, which could result in premature contact of these teeth. This is because the downward-backward rotation of the mandible increases the possibility of an anterior open bite. ${ }^{14,15}$

To our knowledge, no study has evaluated the stability of bimaxillary surgery involving IVRO performed with or without presurgical MARPE in adult patients with skeletal Class 111 malocclusion. Therefore, the purpose of this study was to evaluate differences in postsurgical stability between bimaxillary surgery with MARPE and bimaxillary surgery without MARPE. The null hypothesis was that the stability of bimaxillary surgery is not influenced by expansion of the maxillary arch using MARPE during presurgical orthodontic treatment.

\section{MATERIALS AND METHODS}

\section{Study design and patients}

This retrospective study received institutional review board approval based on the Declaration of Helsinki (22019-0051). Written informed consent was obtained from all patients prior to the initiation of presurgical orthodontic treatment at the Department of Orthodontics, Yonsei University Dental Hospital, Seoul, Republic of Korea.

The subjects were patients who underwent bimaxillary surgery involving IVRO between 2013 and 2017 at the Department of Oral and Maxillofacial Surgery, Yonsei University Dental Hospital, Seoul, Republic of Korea. The patients were divided into control and MARPE groups according to the use of MARPE for nonsurgical maxillary expansion during presurgical orthodontic treatment.

Regardless of the group, the inclusion criteria were as follows: (1) age > 18 years; (2) presence of skeletal Class 111 malocclusion before surgery, with an angle of $<0^{\circ}$ formed by point $A$, the nasion, and point B (ANB); (3) treatment with presurgical orthodontics and conventional bimaxillary surgery involving one-piece le Fort 1 osteotomy and IVRO; (4) use of nonextraction presurgical orthodontic treatment (except extraction of third molars); and (5) availability of a complete series of identifiable lateral cephalograms and dental casts. The exclusion criteria were as follows: (1) history of congenital malformations such as cleft lip and palate and (2) past history of orthognathic surgery and/or orthodontic treatment.

The inclusion criteria for the MARPE group were as follows: (1) transverse maxillary deficiency with unilateral or bilateral posterior crossbite when the maxillary and mandibular posterior teeth were simulated to a Class 1 molar key; (2) a maxillomandibular difference of more than $5 \mathrm{~mm}$ according to Korean standards, ${ }^{16}$ measured on posteroanterior cephalograms before presurgical or- 
thodontics; and (3) midpalatal suture opening confirmed on maxillary anterior periapical radiographs within 4 weeks of activation.

In the MARPE group, rapid maxillary expansion using the MARPE appliance was initiated before orthodontic brackets were bonded (Figure 1). ${ }^{17}$ After being informed about other treatment options such as segmental maxillary osteotomy and SARPE, patients who refused these options due to avoid extended or additional surgery were included. As previously described, ${ }^{8,18}$ a Hyrax screw and four orthodontic miniscrews with a diameter of 1.8 $\mathrm{mm}$ and lengths of $7.0 \mathrm{~mm}$ and $9.0 \mathrm{~mm}$ (self-drilled type, ORLUS; Ortholution, Seoul, Korea) were used for the MARPE appliance. The 9.0- $\mathrm{mm}$ miniscrews were placed in the rugae area while the $7.0-\mathrm{mm}$ screws were placed in the paramidline area of the palate. The heads of the miniscrews were covered by light-cured resin to connect the miniscrews with the helical hooks. The appliance was activated by approximately $0.2 \mathrm{~mm}$ daily until the desired amount of expansion was achieved. ${ }^{19}$

The mean amount of expansion in the maxillary intermolar region was approximately $6.1 \pm 7.2 \mathrm{~mm}$ (mean 30.6 turns; range, 17-40 turns). After a 3-month consolidation period required for bone formation in the median palatal suture, ${ }^{18-20}$ the MARPE appliance was removed and orthodontic brackets were bonded. Presurgical orthodontic treatment was performed for at least 6 months (control group, $11.6 \pm 6.4$ months; MARPE group, $13.0 \pm 4.7$ months), with no statistically significant differences in the treatment duration between the two groups $(p=0.437)$. The patients in the control group received the same presurgical orthodontic treatment, only without MARPE.

\section{Orthognathic treatment}

After presurgical orthodontic treatment, all patients underwent maxillary Le Fort 1 osteotomy and bilateral IVRO performed by a surgeon. Intermaxillary fixation was implemented for 10-14 days after surgery, and physical therapy for rehabilitation was performed for another 2 weeks.

\section{Dental cast analysis}

Dental casts were analyzed using digital calipers to evaluate the changes in tooth positions before presurgical orthodontic treatment (T0) and at 6 months after surgery (T3). The intercanine width (ICW), interpremolar width (IPMW), and intermolar width (IMW) were mea-
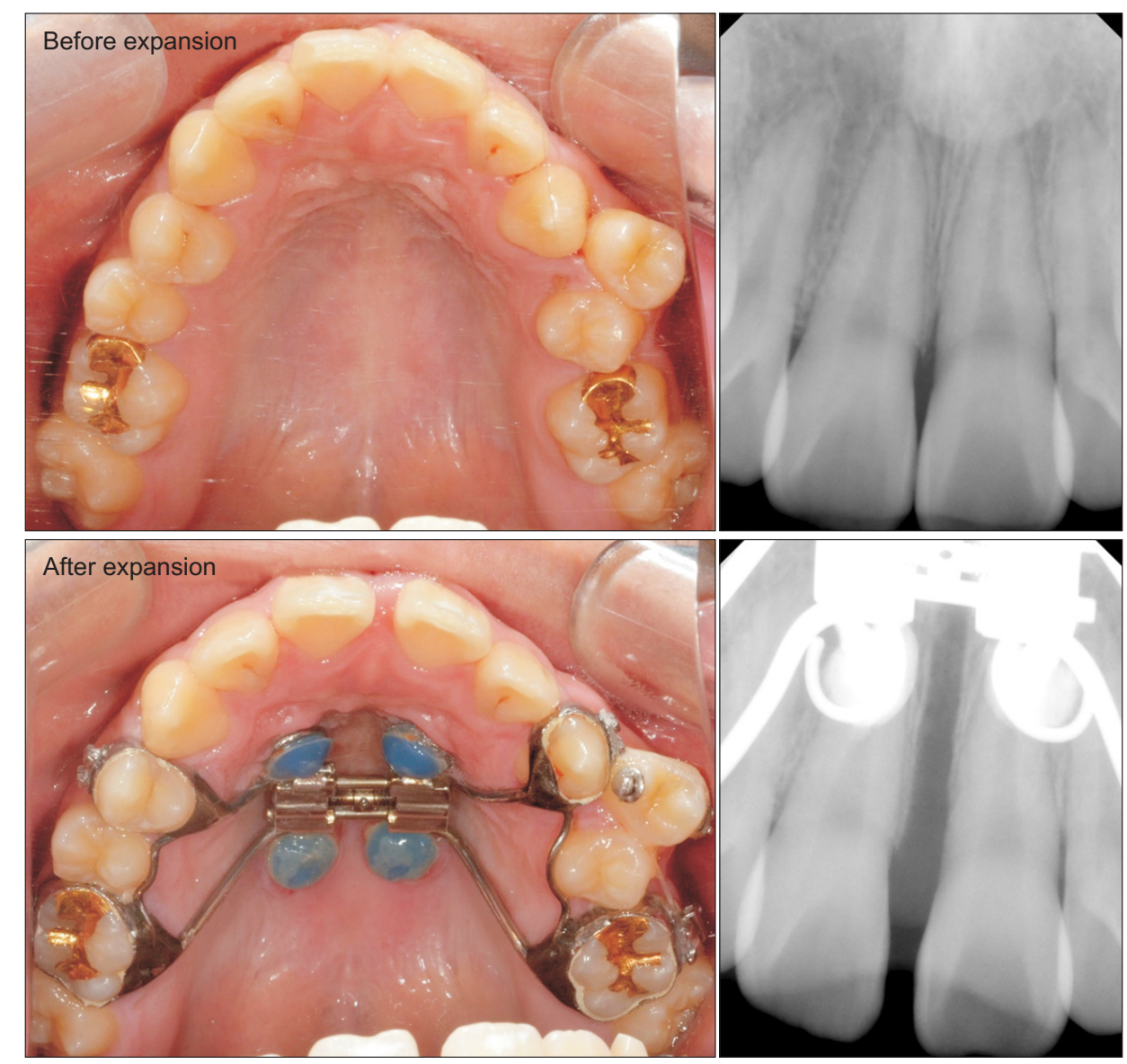

Figure 1. Miniscrew-assisted rapid palatal expansion before bimaxillary surgery. The images show the appliance and periapical radiographs before and after expansion. 
sured. The normal values for differences in the maxillomandibular ICW, IPMW, and IMW in patients with skeletal Class 1 malocclusion are $8.18 \pm 1.57,9.01 \pm 1.66$, and $8.43 \pm 2.22 \mathrm{~mm}$, respectively; this means that the maxillary arch width should exceed the mandibular arch width for ideal occlusion. ${ }^{21}$

\section{Lateral cephalometric analysis}

Skeletal changes were evaluated using lateral cephalograms obtained at 1 month before surgery (T1), 2 days after surgery (T2), and T3. One observer who was blinded to the patient's clinical condition traced the radiographs using V-ceph 5.5 (Osstem, Seoul, Korea). Seven measurements were recorded to analyze the anteroposterior and vertical skeletal changes (Figure 2).

\section{Reliability}

Two weeks after the initial cephalometric and dental cast measurements, all measurements were repeated by the same observer. The method error, calculated using Dahlberg's formula, ranged from 0.20 to $0.30 \mathrm{~mm}$ for linear measurements and from $0.15^{\circ}$ to $0.30^{\circ}$ for angular measurements.

\section{Statistical analysis}

All statistical analyses were performed using IBM SPSS Statistics for Windows, version 22.0 (IBM Corp., Armonk, NY, USA). According to a preliminary study with an effect size of 0.25 for evaluating changes in cephalometric measurements over time in each group, at least 18 subjects were required in each group.

Normality was determined using the Shapiro-Wilk test. The independent $t$-test was used to evaluate the groups at the same time, with the exclusion of data that did not show normality, such as age and sex. Repeated measures analysis of variance was performed to compare skeletal and dental changes over time within and between the two groups. Between-group differences in

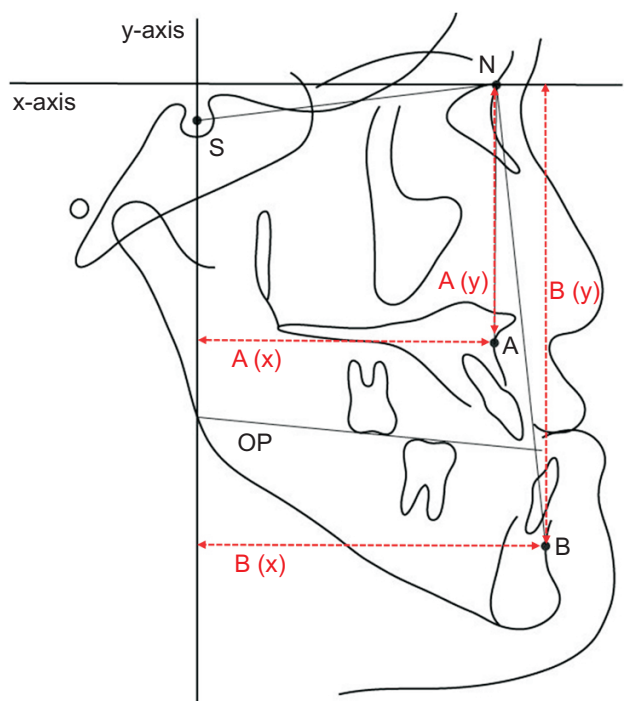

Figure 2. Lateral cephalometric analysis performed to evaluate skeletal changes after bimaxillary surgery with or without presurgical miniscrew-assisted rapid palatal expansion. The $x$-axis is the horizontal plane that shifts the SN plane up $7^{\circ}$ relative to $N$. The $y$-axis is the plane through $S$ and perpendicular to the $x$-axis.

$A$, Point $A ; A(x)$, horizontal position of point $A ; A(y)$, vertical position of point $A ; B$, point $B ; B(x)$, horizontal position of point $B ; B(y)$, vertical position of point $B ; N$, nasion; $\mathrm{OP}$, occlusal plane; $\mathrm{S}$, sella.

Table 1. Baseline characteristics of patients scheduled for bimaxillary surgery with (MARPE group) or without (control group) presurgical MARPE

\begin{tabular}{|c|c|c|c|}
\hline Variable & Control $(n=20)$ & MARPE $(n=20)$ & $p$-value \\
\hline Sex & & & $1.000^{\dagger}$ \\
\hline Male & $10(50.0)$ & $9(45.0)$ & \\
\hline Female & $10(50.0)$ & $11(55.0)$ & \\
\hline Age (yr) & $21.1 \pm 2.6$ & $21.2 \pm 2.9$ & $0.925^{\dagger}$ \\
\hline \multicolumn{4}{|c|}{ Maxillomandibular arch width difference (mm) } \\
\hline ICW difference & $8.0 \pm 2.0$ & $6.7 \pm 2.6$ & $0.089^{\S}$ \\
\hline IPMW difference & $8.0 \pm 2.4$ & $7.4 \pm 3.8$ & $0.608^{\S}$ \\
\hline IMW difference & $6.2 \pm 2.3$ & $3.1 \pm 2.1$ & $<0.001^{\S}$ \\
\hline
\end{tabular}

Values are presented as number (\%) or mean \pm standard deviation.

MARPE, Miniscrew-assisted rapid palatal expansion; ICW, intercanine width; IPMW, interpremolar width; IMW, intermolar width.

${ }^{\dagger}$ The $p$-value was calculated using the chi-squared test.

${ }^{*}$ The $p$-value was calculated using the Mann-Whitney $U$ test.

${ }^{s}$ The $p$-value was calculated using the independent $t$-test. 
Table 2. Changes in maxillary arch widths (T0 to T3) in patients who underwent bimaxillary surgery with (MARPE group) or without (control group) presurgical MARPE

\begin{tabular}{lccc}
\hline \multicolumn{1}{c}{ Variable } & $\begin{array}{c}\text { Control } \\
\text { group }\end{array}$ & $\begin{array}{c}\text { MARPE } \\
\text { group }\end{array}$ & p-value \\
\hline MxICW $(\mathrm{mm})$ & $0.4 \pm 1.5$ & $2.7 \pm 2.1$ & $<0.001$ \\
MxIPMW $(\mathrm{mm})$ & $0.2 \pm 1.2$ & $3.6 \pm 2.4$ & $<0.001$ \\
MxIMW $(\mathrm{mm})$ & $-0.6 \pm 1.2$ & $2.0 \pm 1.3$ & $<0.001$ \\
\hline
\end{tabular}

Values are presented as mean \pm standard deviation.

The $p$-value was calculated using the independent $t$-test.

T0, Before presurgical orthodontic treatment; T3, 6 months after bimaxillary surgery; MARPE, miniscrew-assisted rapid palatal expansion; MxICW, maxillary intercanine width; MxIPMW, maxillary interpremolar width; MxIMW, maxillary intermolar width. changes over time were analyzed using the independent $t$-test with Bonferroni correction $(\alpha=0.05 / 3)$. The correlations of maxillary arch width changes (T3-T0) and surgical changes (T2-T1) with postoperative changes (T3-T2) in all patients were evaluated using the Pearson correlation analysis.

\section{RESULTS}

From 2013 to 2017, 109 patients underwent orthognathic surgery with MARPE. Among these, 32 patients had undergone presurgical orthodontic treatment for < 6 months, 38 patients exhibited menton deviation $>4$ $\mathrm{mm}, 16$ patients exhibited skeletal Class 1 or 11 malocclusion; and three patients did not have their digital cepha-

Table 3. Cephalometric measurements before and after bimaxillary surgery with (MARPE group) or without (control group) presurgical MARPE

\begin{tabular}{|c|c|c|c|c|}
\hline \multirow{2}{*}{ Outcome variable } & \multirow{2}{*}{ Time } & \multirow{2}{*}{ Control group } & \multirow{2}{*}{ MARPE group } & \multirow{2}{*}{$\frac{\text { Time } \times \text { group }}{p \text {-value }}$} \\
\hline & & & & \\
\hline \multirow[t]{3}{*}{ SNA $\left(^{\circ}\right)$} & $\mathrm{T} 1$ & $82.0 \pm 3.0^{\mathrm{BA}}$ & $79.8 \pm 3.3^{\mathrm{BA}}$ & 0.271 \\
\hline & $\mathrm{T} 2$ & $82.3 \pm 3.4^{\mathrm{B}}$ & $80.6 \pm 3.1^{\mathrm{B}}$ & \\
\hline & T3 & $81.5 \pm 3.5^{\mathrm{A}}$ & $80.0 \pm 3.4^{\mathrm{A}}$ & \\
\hline \multirow[t]{3}{*}{$\operatorname{SNB}\left({ }^{\circ}\right)$} & $\mathrm{T} 1$ & $84.4 \pm 3.6^{\mathrm{C}}$ & $81.4 \pm 4.9^{\mathrm{B}}$ & 0.633 \\
\hline & $\mathrm{T} 2$ & $79.0 \pm 3.6^{\mathrm{B}}$ & $76.3 \pm 3.4^{\mathrm{A}}$ & \\
\hline & T3 & $78.2 \pm 3.2^{\mathrm{A}}$ & $75.7 \pm 3.7^{\mathrm{A}}$ & \\
\hline \multirow[t]{3}{*}{ SN-OP $\left(^{\circ}\right)$} & $\mathrm{T} 1$ & $16.8 \pm 3.4^{\mathrm{A}}$ & $19.7 \pm 5.4^{\mathrm{A}}$ & 0.583 \\
\hline & $\mathrm{T} 2$ & $21.4 \pm 5.5^{\mathrm{B}}$ & $25.1 \pm 3.8^{\mathrm{B}}$ & \\
\hline & T3 & $22.3 \pm 4.8^{\mathrm{B}}$ & $26.2 \pm 4.1^{\mathrm{B}}$ & \\
\hline \multirow[t]{3}{*}{$\mathrm{A}(\mathrm{x})(\mathrm{mm})$} & $\mathrm{T} 1$ & $69.5 \pm 5.5^{\mathrm{BA}}$ & $67.3 \pm 4.7^{\mathrm{A}}$ & 0.263 \\
\hline & $\mathrm{T} 2$ & $69.8 \pm 6.1^{\mathrm{B}}$ & $68.0 \pm 4.8^{\mathrm{A}}$ & \\
\hline & $\mathrm{T} 3$ & $68.8 \pm 6.1^{A}$ & $67.4 \pm 5.1^{\mathrm{A}}$ & \\
\hline \multirow[t]{3}{*}{$\mathrm{B}(\mathrm{x})(\mathrm{mm})$} & $\mathrm{T} 1$ & $73.7 \pm 9.4^{\mathrm{C}}$ & $67.7 \pm 10.4^{\mathrm{B}}$ & 0.553 \\
\hline & $\mathrm{T} 2$ & $62.7 \pm 9.2^{\mathrm{B}}$ & $57.4 \pm 7.4^{\mathrm{A}}$ & \\
\hline & $\mathrm{T} 3$ & $61.2 \pm 8.6^{\mathrm{A}}$ & $56.5 \pm 8.5^{\mathrm{A}}$ & \\
\hline \multirow[t]{3}{*}{$\mathrm{A}(\mathrm{y})(\mathrm{mm})$} & $\mathrm{T} 1$ & $68.9 \pm 3.7^{\mathrm{B}}$ & $68.5 \pm 5.1^{A}$ & 0.181 \\
\hline & $\mathrm{T} 2$ & $67.6 \pm 4.7^{\mathrm{BA}}$ & $68.3 \pm 5.3^{\mathrm{A}}$ & \\
\hline & $\mathrm{T} 3$ & $67.0 \pm 3.9^{\mathrm{A}}$ & $67.5 \pm 4.7^{\mathrm{A}}$ & \\
\hline \multirow[t]{3}{*}{ B (y) (mm) } & $\mathrm{T} 1$ & $116.9 \pm 7.4^{\mathrm{B}}$ & $117.0 \pm 8.9^{\mathrm{C}}$ & 0.086 \\
\hline & $\mathrm{T} 2$ & $115.6 \pm 6.1^{\mathrm{BA}}$ & $114.4 \pm 8.0^{\mathrm{B}}$ & \\
\hline & $\mathrm{T} 3$ & $114.5 \pm 6.6^{\mathrm{A}}$ & $113.0 \pm 7.4^{\mathrm{A}}$ & \\
\hline
\end{tabular}

Values are presented as mean \pm standard deviation.

Within each column, significant differences are represented by uppercase letters.

The $p$-value was calculated using repeated measures analysis of variance with Bonferroni correction.

MARPE, Miniscrew-assisted rapid palatal expansion; SNA, angle formed by the lines connecting sella, nasion, and point A; SNB, angle formed by the lines connecting sella, nasion, and point B; SN-OP, angle between the sella-nasion plane and the occlusal plane; A (x), horizontal position of point A; B (x), horizontal position of point B; A (y), vertical position of point A; B (y), vertical position of point $B$; T1, 1 month before surgery; T2, 2 days after surgery; T3, 6 months after surgery. 
lograms or dental casts. Twenty patients were eventually included in the MARPE group.

The control group comprised 10 males and 10 females with an average age of $21.1 \pm 2.6$ years, while the MARPE group comprised nine males and 11 females with an average age of $21.2 \pm 2.9$ years (Table 1). The demographic characteristics before presurgical orthodontic treatment were not significantly different between the two groups. The mean IMW discrepancy was $6.2 \pm$ $2.3 \mathrm{~mm}$ in the control group and $3.1 \pm 2.1 \mathrm{~mm}$ in the MARPE group, with a statistically significant betweengroup difference $(p<0.001)$. This indicated that the maxillary IMW in patients in the MARPE group was approximately $3.1 \mathrm{~mm}$ lesser than that in patients in the control group.

Table 2 shows that all maxillary arch width changes (i.e., T3-T0), measured on the maxillary dental casts, were significantly different between the two groups $(p<$ $0.001)$. In the MARPE group, the maxillary ICW $(2.7 \pm 2.1$ $\mathrm{mm})$, IPMW $(3.6 \pm 2.4 \mathrm{~mm})$, and IMW $(2.0 \pm 1.3 \mathrm{~mm})$ were significantly increased $(p<0.001)$ relative to the values before presurgical orthodontic treatment. In the control group, however, the widths were maintained or even decreased.

Table 3 shows that all cephalometric measurements at the different time points were not significantly different between the two groups. At T1, T2, and T3 in the MARPE group, point A was located $68.5 \pm 5.1 \mathrm{~mm}, 68.3$ $\pm 5.3 \mathrm{~mm}$, and $67.5 \pm 4.7 \mathrm{~mm}$ vertically on the x-axis, respectively. However, the values were not significantly different.
From T1 to T2, point B moved $10.9 \pm 3.7 \mathrm{~mm}$ posterior $(p<0.001)$ and $1.5 \pm 2.3 \mathrm{~mm}$ superior in the control group and $10.3 \pm 5.2 \mathrm{~mm}$ posterior $(p<0.001)$ and $3.9 \pm 3.5 \mathrm{~mm}$ superior $(p<0.001)$ in the MARPE group (Table 4). However, none of the surgical changes from $\mathrm{T} 1$ to $\mathrm{T} 2$ showed significant differences between the two groups.

During the postsurgical period (T2 to T3), the angle between the sella-nasion plane and the occlusal plane increased by $0.8 \pm 2.3^{\circ}$ in the control group and $1.0 \pm$ $2.0^{\circ}$ in the MARPE group; however, this angular change was not significant within each group or between the two groups (Table 5). Point B moved $1.5 \pm 1.7 \mathrm{~mm}$ backward $(p=0.003)$ and $1.0 \pm 2.1 \mathrm{~mm}$ upward in the control group and $0.8 \pm 2.0 \mathrm{~mm}$ backward and $1.3 \pm 1.6$ mm upward $(p=0.004)$ in the MARPE group. However, the two groups showed no significant differences in the postoperative changes in all measurements over time.

In both groups, the amount of upward mandibular movement at 6 months after surgery (T2 to T3) decreased as the amount of upward mandibular movement during surgery ( $\mathrm{T} 1$ to $\mathrm{T} 2$ ) increased $(r=-0.330 ; p=0.038$; Table 6). However, the amounts of horizontal mandibular movements during surgery (T1 to T2) and the amount of expansion in the maxillary intermolar region during the overall treatment period (T0 to T3) showed no significant correlation with the postsurgical changes in the mandible (T2 to T3).

Table 4. Surgical changes (T1 to T2) in cephalometric measurements for patients who underwent bimaxillary surgery with (MARPE group) or without (control group) presurgical MARPE

\begin{tabular}{|c|c|c|c|c|c|}
\hline \multirow{2}{*}{ T2-T1 } & \multicolumn{2}{|c|}{ Control group } & \multicolumn{2}{|c|}{ MARPE group } & \multirow{2}{*}{ Between groups } \\
\hline & Difference & $p$-value & Difference & $p$-value & \\
\hline SNA $\left(^{\circ}\right)$ & $0.2 \pm 1.6$ & 1.000 & $0.7 \pm 1.2$ & 0.052 & 0.286 \\
\hline $\operatorname{SNB}\left({ }^{\circ}\right)$ & $-5.4 \pm 1.9$ & $<0.001$ & $-5.0 \pm 2.7$ & $<0.001$ & 0.614 \\
\hline $\mathrm{SN}-\mathrm{OP}\left({ }^{\circ}\right)$ & $4.6 \pm 3.6$ & $<0.001$ & $5.4 \pm 3.1$ & $<0.001$ & 0.502 \\
\hline $\mathrm{A}(\mathrm{x})(\mathrm{mm})$ & $0.2 \pm 1.6$ & 1.000 & $0.7 \pm 1.7$ & 0.264 & 0.405 \\
\hline $\mathrm{B}(\mathrm{x})(\mathrm{mm})$ & $-10.9 \pm 3.7$ & $<0.001$ & $-10.3 \pm 5.2$ & $<0.001$ & 0.647 \\
\hline $\mathrm{A}(\mathrm{y})(\mathrm{mm})$ & $-1.3 \pm 2.4$ & 0.076 & $-0.1 \pm 2.0$ & 1.000 & 0.113 \\
\hline B (y) (mm) & $-1.5 \pm 2.3$ & 0.319 & $-3.9 \pm 3.5$ & $<0.001$ & 0.125 \\
\hline
\end{tabular}

Values are presented as mean \pm standard deviation.

Group comparisons were tested using the independent $t$-test with Bonferroni correction $(p<0.05 / 3)$. Positive and negative values indicate, respectively, the anterior and posterior horizontal changes, inferior and superior vertical changes, and increased and decreased dimensional changes.

The $p$-value was calculated using repeated measures analysis of variance with Bonferroni correction.

T1, 1 month before surgery; T2, 2 days after surgery; MARPE, miniscrew-assisted rapid palatal expansion; SNA, angle formed by the lines connecting the sella, nasion, and point $\mathrm{A}$; SNB, angle formed by the lines connecting the sella, nasion, and point $\mathrm{B}$; SN-OP, angle between the sella-nasion plane and the occlusal plane; A (x), horizontal position of point A; B (x), horizontal position of point B; A (y), vertical position of point A; B (y), vertical position of point B. 
Table 5. Postoperative relapse (T2 to T3) in cephalometric measurements for patients who underwent bimaxillary surgery with (MARPE group) or without (control group) presurgical MARPE

\begin{tabular}{lcccccc}
\hline \multirow{2}{*}{ T3-T2 } & \multicolumn{2}{c}{ Control group } & & \multicolumn{2}{c}{ MARPE group } & \multirow{2}{*}{ Between groups } \\
\cline { 2 - 3 } \cline { 5 - 6 } & Difference & $\boldsymbol{p}$-value & & Difference & $\boldsymbol{p}$-value & 0.029 \\
SNA $\left(^{\circ}\right)$ & $-0.7 \pm 0.7$ & 0.001 & & $-0.5 \pm 0.9$ & 0.475 \\
SNB $\left(^{\circ}\right)$ & $-0.7 \pm 0.8$ & 0.002 & & $-0.5 \pm 1.1$ & 0.133 & 0.485 \\
SN-OP $\left(^{\circ}\right)$ & $0.8 \pm 2.3$ & 0.342 & & $1.0 \pm 2.0$ & 0.103 & 0.780 \\
A (x) (mm) & $-0.9 \pm 1.0$ & 0.002 & & $-0.5 \pm 1.0$ & 0.075 & 0.256 \\
B (x) (mm) & $-1.5 \pm 1.7$ & 0.003 & & $-0.8 \pm 2.0$ & 0.215 & 0.268 \\
A (y) (mm) & $-0.5 \pm 1.8$ & 0.698 & & $-0.8 \pm 1.5$ & 0.096 & 0.584 \\
B (y) (mm) & $-1.0 \pm 2.1$ & 0.116 & & $-1.3 \pm 1.6$ & 0.004 & 0.622 \\
\hline
\end{tabular}

Values are presented as mean \pm standard deviation.

Group comparisons were tested using the independent $t$-test with Bonferroni correction $(p<0.05 / 3)$. The positive and negative values indicate, respectively, the anterior and posterior horizontal changes, inferior and superior vertical changes, and increased and decreased dimensional changes.

The $p$-value was calculated using repeated measures analysis of variance with Bonferroni correction.

T2, 2 days after surgery; T3, 6 months after surgery; MARPE, miniscrew-assisted rapid palatal expansion; SNA, angle formed by the lines connecting the sella, nasion, and point $\mathrm{A}$; SNB, angle formed by the lines connecting the sella, nasion, and point $\mathrm{B}$; SN-OP, angle between the sella-nasion plane and the occlusal plane; A (x), horizontal position of point A; B (x), horizontal position of point $B ; A(y)$, vertical position of point A; B (y), vertical position of point $B$.

Table 6. Correlations of maxillary arch width changes (T3-T0) and surgical changes (T2-T1) with postoperative changes (T3-T2) in patients who underwent bimaxillary surgery with (MARPE group) or without (control group) presurgical MARPE

\begin{tabular}{|c|c|c|c|c|}
\hline \multirow{3}{*}{ Variable } & \multicolumn{4}{|c|}{ Postsurgical change 6 months after surgery (T3-T2) } \\
\hline & \multicolumn{2}{|c|}{ B (x) (mm) } & \multicolumn{2}{|c|}{$B(y)(\mathrm{mm})$} \\
\hline & $\mathbf{r}$ & $p$-value & $\boldsymbol{r}$ & $p$-value \\
\hline MxICW (T3-T0) (mm) & 0.185 & 0.254 & -0.155 & 0.339 \\
\hline MxIPMW (T3-T0) (mm) & 0.120 & 0.460 & 0.032 & 0.845 \\
\hline MxIMW (T3-T0) (mm) & -0.068 & 0.677 & 0.208 & 0.198 \\
\hline $\mathrm{B}(\mathrm{x})(\mathrm{T} 2-\mathrm{T} 1)(\mathrm{mm})$ & -0.221 & 0.170 & 0.193 & 0.233 \\
\hline B (y) (T2-T1) (mm) & -0.027 & 0.868 & -0.330 & 0.038 \\
\hline
\end{tabular}

The $p$-value was calculated using Pearson correlation analysis.

T0, Before presurgical orthodontics; T1, 1 month before surgery; T2, 2 days after surgery; T3, 6 months after surgery; MARPE, miniscrew-assisted rapid palatal expansion; B (x), horizontal position of point B; B (y), vertical position of point B; $r$, Pearson's correlation coefficient; MxICW, maxillary intercanine width; MxIPMW, maxillary interpremolar width; MxIMW, maxillary intermolar width.

\section{DISCUSSION}

Nonsurgical maxillary expansion using MARPE leads to a pyramidal pattern of changes in the circummaxillary structures, the center of rotation of which is located near the frontonasal suture. ${ }^{22} \mathrm{Lim}$ et $\mathrm{al} .{ }^{20}$ investigated the stability of the treatment outcome at 1 year after MARPE expansion and reported that more than half of the expansion occurred in the dentoalveolar portion. Hong $^{23}$ reported that the axial angulation of the maxillary first molar in adults increased by $2.29 \pm 8.09^{\circ}$ upon removal of the fixed orthodontic appliance after completion of nonsurgical orthodontic treatment with MARPE; this indicated that the maxillary molar is slightly buccally inclined after orthodontic treatment. In view of this finding, relapse of the tilted molars and alveolar bone after MARPE could cause occlusal interferences in the postsurgical period. Previous studies ${ }^{13,24}$ have reported posterior and inferior relapse with clockwise rotation of the mandible after setback surgery using IVRO. Because IVRO does not involve rigid fixation between the proximal and distal segments immediately after surgery, con- 
siderable caution should be exercised to prevent anterior open bite due to temporary condylar sagging or muscular pull of the masticatory muscles up to 3 months after surgery. Considering these two factors (i.e., relapse of maxillary constriction and mandibular vertical instability with IVRO), we were skeptical about the results of mandibular setback by IVRO combined with nonsurgical maxillary expansion using MARPE in patients with skeletal Class 111 malocclusion and transverse maxillary deficiency.

Before presurgical orthodontic treatment, the MARPE group exhibited a greater arch width discrepancy in the molar region than did the control group. From T0 to T3, the maxillary IMW was significantly increased in the MARPE group $(2.0 \pm 1.3 \mathrm{~mm})$, but not in the control group $(-0.6 \pm 1.2 \mathrm{~mm})$. The mean amount of expansion with the MARPE appliance was approximately $6 \mathrm{~mm}(30$ turns $\times 0.2 \mathrm{~mm}$ per turn) in this study, although the actual remaining amount of expansion at T3 was approximately $2 \mathrm{~mm}$ in the molar region. These phenomena may be attributed to lingual repositioning of the molars during the alignment phase, depending on the arch form during the pre- and postsurgical orthodontic treatments. ${ }^{18}$ This factor was considered before surgery, and the maxilla was over-expanded using MARPE until the palatal cusps of the maxillary posterior teeth contacted the buccal cusps of the mandibular posterior teeth, similar to the outcome after conventional RPE.

Handelman et al..$^{25}$ stated that SARPE should be used when the required increase in the maxillary IMW exceeds $8 \mathrm{~mm}$. Choi et al. ${ }^{18}$ stated that MARPE caused skeletal expansion that is approximately $43 \%$ of the maxillary IMW expansion, unlike appliances that induce buccal tilting of the molars, such as the transpalatal arch. Therefore, according to the results of this study, we recommend the use of MARPE in cases where the maxillomandibular IMW difference is approximately 3-4 $\mathrm{mm}$ smaller than the normal value than wire expansion with a transpalatal arch or arch width control by maxillary premolar extraction. This is because MARPE opens the midpalatal suture, inducing bone formation between the separated sutures that ensures maintenance of the maxillary arch width even after the molars are lingually uprighted due to the intrinsic buccolingual inclination of the brackets during presurgical orthodontics. ${ }^{18-20,26}$

In several previous studies, ${ }^{27-29}$ skeletal tissues have been reported to be stable at 6 months after orthognathic surgery. Normally, debonding in cases undergoing presurgical orthodontic treatment is performed within 6 months of surgery. Accordingly, we also followed our cases for 6 months after surgery. Point A did not show significant anteroposterior or vertical movement at $\mathrm{T} 1$, T2, and T3 in the MARPE group. $\mathrm{Hong}^{23}$ reported that maxillary expansion using MARPE in adults induced forward and downward movement of the maxilla, with no change in the tilt of the palatal plane relative to the cranial base. This displaced maxillary position is reportedly maintained after debonding. In the present study, forward and downward movements of the maxilla may have occurred during presurgical orthodontic treatment with MARPE; however, the position of point A was relatively stable between 2 days and 6 months after orthognathic surgery. This indicates that the maxilla was anteroposteriorly and vertically stable after the surgery.

Two days after surgery, there were no significant intergroup differences in the amount of surgical movement of point B in any direction. At 6 months after surgery, the stability was not different between the two groups. Both groups showed backward and upward mandibular movement during surgery which was also seen after surgery. However, some of the latter changes were not insignificant. The postsurgical backward movement of the mandible was consistent with the findings of some previous studies performed using the IVRO technique. ${ }^{13,30}$ In addition, no significant correlation existed between the amount of maxillary expansion and the postsurgical movement of the mandible.

This study has many limitations. First, it was a retrospective study. Second, only two-dimensional radiographs were analyzed. Third, the sample size was small, although it did not affect the statistical analyses. Therefore, it is difficult to make a generalization based on the results of this study. However, the outcomes at 6 months after bimaxillary surgery, including mandibular setback by IVRO, did not differ significantly, regardless of the use of MARPE before surgery. These findings legitimize the use of nonsurgical maxillary expansion using MARPE and the IVRO technique in patients with skeletal Class 111 malocclusion and transverse maxillomandibular discrepancy.

\section{CONCLUSION}

The findings of this study suggest that MARPE is useful for stable and nonsurgical expansion of the maxilla in adult patients with skeletal Class 111 malocclusion and transverse maxillary deficiency who are scheduled for bimaxillary surgery.

\section{CONFLICTS OF INTEREST}

No potential conflict of interest relevant to this article was reported.

\section{ACKNOWLEDGEMENTS}

This research was partially supported by the Basic Science Research Program through the National Re- 
search Foundation of Korea (NRF) funded by the Ministry of Science, ICT and Future Planning (NRF2018R1C1B6000989).

\section{REFERENCES}

1. Ahn J, Kim SJ, Lee JY, Chung CJ, Kim KH. Transverse dental compensation in relation to sagittal and transverse skeletal discrepancies in skeletal Class 111 patients. Am J Orthod Dentofacial Orthop 2017;151:148-56.

2. Proffit WR, Turvey TA, Phillips C. The hierarchy of stability and predictability in orthognathic surgery with rigid fixation: an update and extension. Head Face Med 2007;3:21.

3. Kim H, Cha KS. Evaluation of the stability of maxillary expansion using cone-beam computed tomography after segmental Le Fort 1 osteotomy in adult patients with skeletal Class 111 malocclusion. Korean J Orthod 2018;48:63-70.

4. Dergin G, Aktop S, Varol A, Ugurlu F, Garip H. Complications related to surgically assisted rapid palatal expansion. Oral Surg Oral Med Oral Pathol Oral Radiol 2015;119:601-7.

5. Haas Junior OL, Guijarro-Martínez R, de Sousa Gil AP, da Silva Meirelles L, de Oliveira RB, HernándezAlfaro F. Stability and surgical complications in segmental Le Fort 1 osteotomy: a systematic review. Int J Oral Maxillofac Surg 2017;46:1071-87.

6. Lee SJ, Kim TW, Nahm DS. Transverse implications of maxillary premolar extraction in Class 111 presurgical orthodontic treatment. Am J Orthod Dentofacial Orthop 2006;129:740-8.

7. Persson M, Thilander B. Palatal suture closure in man from 15 to 35 years of age. Am J Orthod 1977; 72:42-52.

8. Lee KJ, Park YC, Park JY, Hwang WS. Miniscrew-assisted nonsurgical palatal expansion before orthognathic surgery for a patient with severe mandibular prognathism. Am J Orthod Dentofacial Orthop 2010;137:830-9.

9. Seong EH, Choi SH, Kim HJ, Yu HS, Park YC, Lee KJ. Evaluation of the effects of miniscrew incorporation in palatal expanders for young adults using finite element analysis. Korean J Orthod 2018;48:81-9.

10. Chung CH, Woo A, Zagarinsky J, Vanarsdall RL, Fonseca RJ. Maxillary sagittal and vertical displacement induced by surgically assisted rapid palatal expansion. Am J Orthod Dentofacial Orthop 2001;120:144-8.

11. Chung $\mathrm{CH}$, Font B. Skeletal and dental changes in the sagittal, vertical, and transverse dimensions after rapid palatal expansion. Am J Orthod Dentofacial Orthop 2004;126:569-75.
12. Habeeb M, Boucher N, Chung CH. Effects of rapid palatal expansion on the sagittal and vertical dimensions of the maxilla: a study on cephalograms derived from cone-beam computed tomography. Am J Orthod Dentofacial Orthop 2013;144:398-403.

13. Yoshioka 1, Khanal A, Tominaga K, Horie A, Furuta N, Fukuda J. Vertical ramus versus sagittal split osteotomies: comparison of stability after mandibular setback. J Oral Maxillofac Surg 2008;66:1138-44.

14. Suri L, Taneja P. Surgically assisted rapid palatal expansion: a literature review. Am J Orthod Dentofacial Orthop 2008;133:290-302.

15. Akkaya S, Lorenzon S, Uçem TT. A comparison of sagittal and vertical effects between bonded rapid and slow maxillary expansion procedures. Eur J Orthod 1999;21:175-80.

16. Hwang S, Noh Y, Choi YJ, Chung C, Lee HS, Kim $\mathrm{KH}$. Dentofacial transverse development in Koreans according to skeletal maturation: a cross-sectional study. Korean J Orthod 2018;48:39-47.

17. Lee KJ, Choi SH, Choi TH, Shi KK, Keum BT. Maxillary transverse expansion in adults: rationale, appliance design, and treatment outcomes. Semin Orthod 2018;24:52-65.

18. Choi SH, Shi KK, Cha JY, Park YC, Lee KJ. Nonsurgical miniscrew-assisted rapid maxillary expansion results in acceptable stability in young adults. Angle Orthod 2016;86:713-20.

19. Park JJ, Park YC, Lee KJ, Cha JY, Tahk JH, Choi YJ. Skeletal and dentoalveolar changes after miniscrewassisted rapid palatal expansion in young adults: a cone-beam computed tomography study. Korean J Orthod 2017;47:77-86.

20. Lim HM, Park YC, Lee KJ, Kim KH, Choi YJ. Stability of dental, alveolar, and skeletal changes after miniscrew-assisted rapid palatal expansion. Korean J Orthod 2017;47:313-22.

21. Koo YJ, Choi SH, Keum BT, Yu HS, Hwang CJ, Melsen B, et al. Maxillomandibular arch width differences at estimated centers of resistance: comparison between normal occlusion and skeletal Class 111 malocclusion. Korean J Orthod 2017;47:167-75.

22. Park JC, Baek WS, Choi SH, Cho KS, Jung UW. Longterm outcomes of dental implants placed in elderly patients: a retrospective clinical and radiographic analysis. Clin Oral Implants Res 2017;28:186-91.

23. Hong HG. Changes in sagittal and vertical dimensions by non-surgical miniscrew-assisted rapid palatal expansion in adults [MD thesis]. Seoul: Yonsei University; 2019.

24. Nihara J, Takeyama M, Takayama Y, Mutoh Y, Saito 1. Postoperative changes in mandibular prognathism surgically treated by intraoral vertical ramus osteotomy. Int J Oral Maxillofac Surg 2013;42:62-70. 
25. Handelman CS, Wang L, BeGole EA, Haas AJ. Nonsurgical rapid maxillary expansion in adults: report on 47 cases using the Haas expander. Angle Orthod 2000;70:129-44.

26. Shin $\mathrm{H}$, Hwang $\mathrm{CJ}$, Lee KJ, Choi YJ, Han SS, Yu HS. Predictors of midpalatal suture expansion by miniscrew-assisted rapid palatal expansion in young adults: a preliminary study. Korean J Orthod 2019;49:360-71.

27. Han JJ, Yang HJ, Lee SJ, Hwang SJ. Relapse after SSRO for mandibular setback movement in relation to the amount of mandibular setback and intraoperative clockwise rotation of the proximal segment. J Craniomaxillofac Surg 2014;42:811-5.

28. Souza Pinto GN, Iwaki Filho L, Previdelli ITDS, Ra- mos AL, Yamashita AL, Stabile GAV, et al. Threedimensional alterations in pharyngeal airspace, soft palate, and hyoid bone of class 11 and class 111 patients submitted to bimaxillary orthognathic surgery: a retrospective study. J Craniomaxillofac Surg 2019;47:883-94.

29. Park KH, Sandor GK, Kim YD. Skeletal stability of surgery-first bimaxillary orthognathic surgery for skeletal class 111 malocclusion, using standardized criteria. Int J Oral Maxillofac Surg 2016;45:35-40.

30. Jung HD, Jung YS, Kim SY, Kim DW, Park HS. Postoperative stability following bilateral intraoral vertical ramus osteotomy based on amount of setback. Br J Oral Maxillofac Surg 2013;51:822-6. 